\title{
Demonstrate Benchtop Measurement of Recrystallization
}

\author{
David Hurley \\ Stephen Reese
}

September 2017

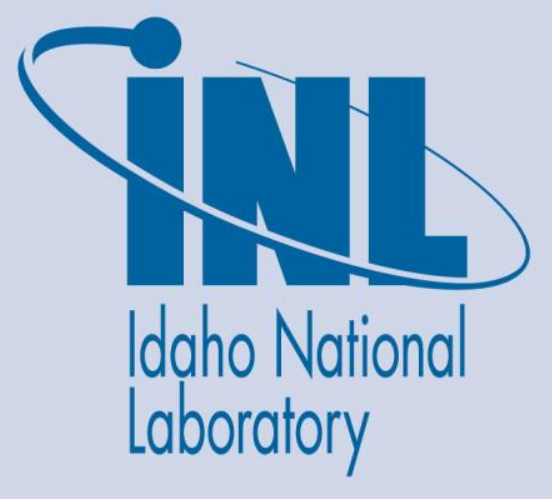

The INL is a U.S. Department of Energy National Laboratory operated by Battelle Energy Alliance 


\section{DISCLAIMER}

This information was prepared as an account of work sponsored by an agency of the U.S. Government. Neither the U.S. Government nor any agency thereof, nor any of their employees, makes any warranty, expressed or implied, or assumes any legal liability or responsibility for the accuracy, completeness, or usefulness, of any information, apparatus, product, or process disclosed, or represents that its use would not infringe privately owned rights. References herein to any specific commercial product, process, or service by trade name, trade mark, manufacturer, or otherwise, does not necessarily constitute or imply its endorsement, recommendation, or favoring by the U.S. Government or any agency thereof. The views and opinions of authors expressed herein do not necessarily state or reflect those of the U.S. Government or any agency thereof. 


\title{
Demonstrate Benchtop Measurement of Recrystallization
}

\author{
David Hurley \\ Stephen Reese
}

September 2017

Idaho National Laboratory Idaho Falls, Idaho 83415

http://www.inl.gov

\author{
Prepared for the \\ U.S. Department of Energy \\ Office of Nuclear Energy \\ Under DOE Idaho Operations Office \\ Contract DE-AC07-05ID14517
}





\begin{abstract}
The Mechanical Properties Microscope (MPM) is used to monitor grain restructuring (recrystallization) of a metallic fuel surrogate sample. The MPM is a laser ultrasonic instrument used to measure the elastic and anelastic response of a material. The MPM has been used previously to measure the elastic constants of a $\mathrm{U} \cdot \mathrm{Zr}$ system both as a function of composition and temperature. In this study we extend the capability of the MPM to make measurements of the resonant response of a small metallic fuel surrogate as a function of temperature. The fuel surrogate consists of a highly textured copper sample. By tracking specific resonant modes as a function of temperature we demonstrate that the MPM is well suited to measure grain restructuring in metallic fuel surrogates.
\end{abstract}




\section{CONTENTS}

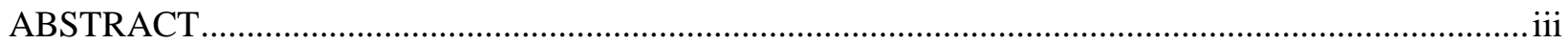

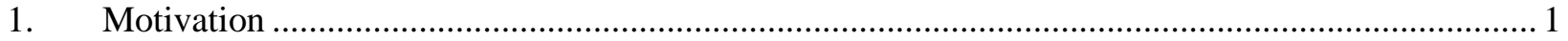

2. Indirect Measurement of Microstructure using Ultrasound........................................................ 1

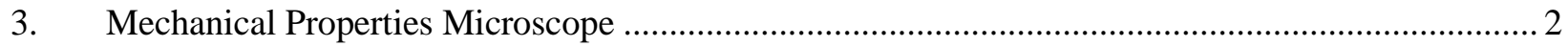

4. Extending the MPM's Capability to Measure Grain Restructuring in a Metallic Fuel

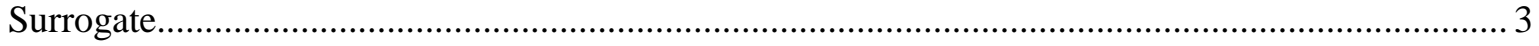

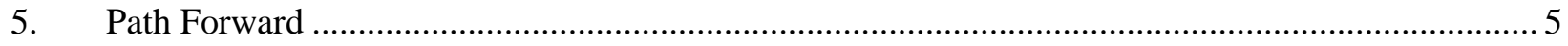

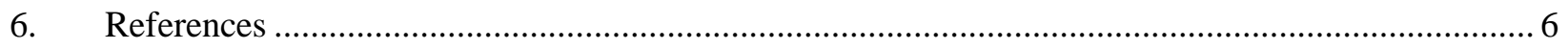

FIGURES

Figure 1. The two modes of operation for the MPM. In the time domain the MPM can be used to measure elastic constants. The frequency domain is ideally suited for ultrasonic

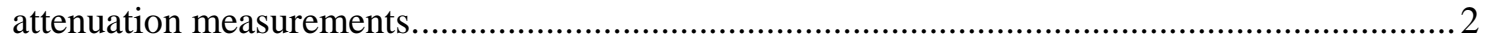

Figure 2. Resonant mode frequencies before (blue) and after (red) recrystallization. The insets show the resonant mode shapes before (R1 - R5, R11, R12) and after (A1 - A4, A6, A15, A16) recrystallization.

Figure 3. Tracks of individual modes throughout the heating and cooling process. Note how modes R1 and R2 and modes R3 and R4 change position relative to one another 


\section{Demonstrate Benchtop Measurement of Recrystallization}

\section{Motivation}

Microstructure evolution in nuclear fuel is governed by atomic transport facilitated by a highly nonequilibrium distribution of Frenkel defects. The interstitial portion of Frenkel defects are preferentially drawn to dislocations, causing dislocations to multiply. The strain energy associated with dislocation production coupled with high temperatures cause profound changes to the grain microstructure. Grain restructuring or recrystallization occurs in both metal and ceramic nuclear fuel. Grain restructuring can result in large changes in texture that will alter both the elastic and plastic properties of fuel. In ceramic fuel, grain refinement in the high burnup rim structure leads to small crystallites having diameters of a few hundred nanometers. In the rim region the large boundary area to volume ratio significantly influences defect accumulation near the boundaries and also impacts thermal transport.

\section{Indirect Measurement of Microstructure using Ultrasound}

Ultrasonics holds great potential to indirectly measure microstructure in an in-pile environment. This approach involves relating the constitutive relation between stress and strain to microstructure. A typical experiment measures either ultrasonic velocity or attenuation. For instance, measuring the resonant frequency of a long narrow bar yields an expression for the modulus of elasticity, E. Measurement of the bulk longitudinal velocity leads to an expression that contains E and Poisson's ratio, $v$. For nonattenuative, isotropic materials, these two elastic constants define the complete stress-strain relationship. However, all materials exhibit attenuation and are rarely isotropic. Departure from isotropy requires more elastic constants to define the stress-strain relation. For instance, a polycrystalline metal plate that has been textured by rolling requires nine elastic constants to define the stress-strain relation. For attenuative materials, in which some ultrasonic energy is converted into another form of energy, the ultrasonic velocities, in general, are functions of frequency. In this case, the relation between stress and strain is referred to as viscoelasticity.

Large microstructure features, like voids/pores, have a strong influence on ultrasonic velocity. This has led researchers to explore the relation between changes in velocity and changes in swelling in thickwalled reactor vessel components. This characterization approach is able to map spatial variations in swelling. Changes in texture caused by deformation-induced recrystallization can also dramatically influence velocity. Similar to deformation driven processes, irradiation induced dislocation growth causes nuclear fuel to recrystallize and/or restructure. Ultrasonic attenuation can also be related to changes in microstructure. Using Granato Lucke theory, ultrasonic attenuation can be related to dislocation line length and density.

In principle, the concept of relating constitutive relations to microstructure is easy to comprehend; however, in practice there are several issues that need to be addressed to accurately and uniquely make this connection. First the size of the effect must be considered. For instance, attenuation due to irradiation induced dislocation growth can be orders of magnitude smaller than attenuation caused by slight changes in texture. Thus, accurately associating changes in dislocation density and pinning length to ultrasonic attenuation will require the use of single crystal samples. Attenuation due to ultrasonic coupling to the environment can also overwhelm the contribution from dislocations. Accounting for this source of apparent attenuation requires designing experiments where the sample is ultrasonically isolated from the environment. Changing coupling conditions associated with contract transduction methods can also lead to issues associated with measurement reproducibility. 
Other issues are inherent to ultrasonics and are related to the physics of wave mechanics. All ultrasonic waves experience diffraction, i.e., spreading of the wave front. This gives rise to another source of attenuation called geometric attenuation. Correctly accounting for this source of attenuation requires confining the ultrasonic energy to a particular geometry. This approach, termed resonant ultrasound spectroscopy (RUS), involves measuring the mechanical resonances of an ultrasonically isolated sample. Another issue related to wave mechanics is frequency dependent dispersion. For wave guiding geometries, such as long narrow bars or plates, the ultrasonic velocity is a strong function of wavelength, when the wavelength is comparable to the sample dimensions. Wave guiding geometries can be readily modeled; however, this does add another layer of complication that should be avoided if possible.

\section{Mechanical Properties Microscope}

The Mechanical Properties Microscope was developed previously under the Fuel Cycle Research and Development Program. The MPM uses a pulsed laser to thermoelastically excite ultrasonic waves in a sample and a laser interferometer to detect out-of-plane displacements associated with the propagating ultrasonic waves. The MPM has two modes of operation.

In the time domain the MPM can be used to measure bulk waves, surface waves, or guided waves.i An example of a typical bulk wave measurement is shown in the top pane of Figure 1. The experimental geometry involves point excitation and backside detection along the epicentral axis. The arrival of the longitudinal wave is denoted by a step change in the wave form (at $\sim 560 \mathrm{~ns}$ in the graph on the right side of the figure) and the shear wave arrival is denoted by a trough (at $\sim 1000 \mathrm{~ns}$ in the figure). Also shown on the graph is a Green's function solution for a laser point source in an elastically isotropic material. The Green's function plot (smooth, bold line) is superimposed on the experimentally measured waveform (noisier, gray line). The elastic constants can be obtained by minimizing the standard deviation between experiment and theory.

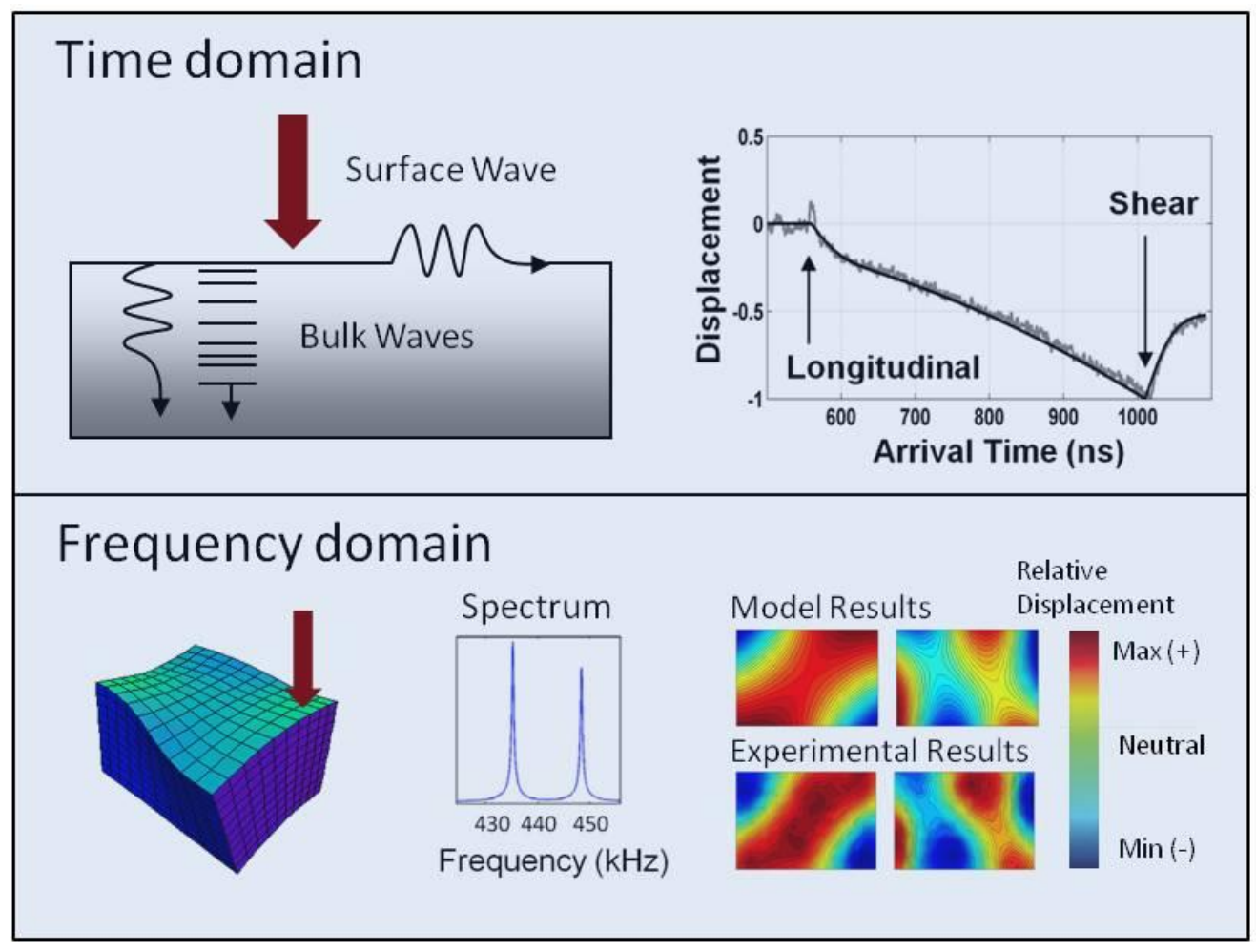

Figure 1. The two modes of operation for the MPM. In the time domain the MPM can be used to measure elastic constants. The frequency domain is ideally suited for ultrasonic attenuation measurements. 
In the frequency domain the MPM excites broadband resonances in a sample with known density and dimensions (the bottom pane of Figure 1). The eigenfrequencies are obtained by measuring the vibration ring-down and taking a Fourier transform of the time domain response. The eigenmodes (i.e., out-of-plane displacement as a function of position along the sample surface) are obtained by windowing a particular resonant peak and measuring amplitude variation with probe scan position.ii The frequency domain approach is ideally suited for ultrasonic attenuation measurements. In addition, low symmetry materials do not add complication to performing the forward problem of predicting the eigenfrequencies from the elastic stiffness tensor.

\section{Extending the MPM's Capability to Measure Grain Restructuring in a Metallic Fuel Surrogate}

Laser-based resonant ultrasound spectroscopy was employed to monitor recrystallization in a high purity copper parallelepiped sample, measuring $\sim 5.4 \times 3.3 \times 0.5 \mathrm{~mm}$. The sample had been previously rolled to imbue a texture in it. To demonstration the ability to monitor recrystallization using RUS, three sets of measurements were taken on the sample. First, the sample was scanned in a $17 \times 9$ grid. The sample's resonant response was captured at each of the 153 points. From these spectra, the shapes (eigenmodes) and frequencies (eigenvalues) of the sample's resonant modes were determined. Next, the sample was annealed in a furnace - heating from room temperature to $300^{\circ} \mathrm{C}$, and then cooling back to room temperature. Resonant response was measured in situ at 32 temperature steps. The in situ measurements were made at a single location on the $17 \times 9$ grid previously scanned. Finally, the entire 17 $\times 9$ grid was rescanned at room temperature to capture the frequencies and shapes of the resonant modes of the now annealed sample.

With knowledge of the mode shapes, how individual modes' frequencies change as the material transforms from a rolled texture to an annealed state can be tracked. To demonstrate this concept, seven resonant modes were tracked throughout the recrystallization process. Figure 2 shows a plot of the sample's resonant response before recrystallization (rolled) and after (annealed). The insets show the mode shapes of the seven tracked modes. 


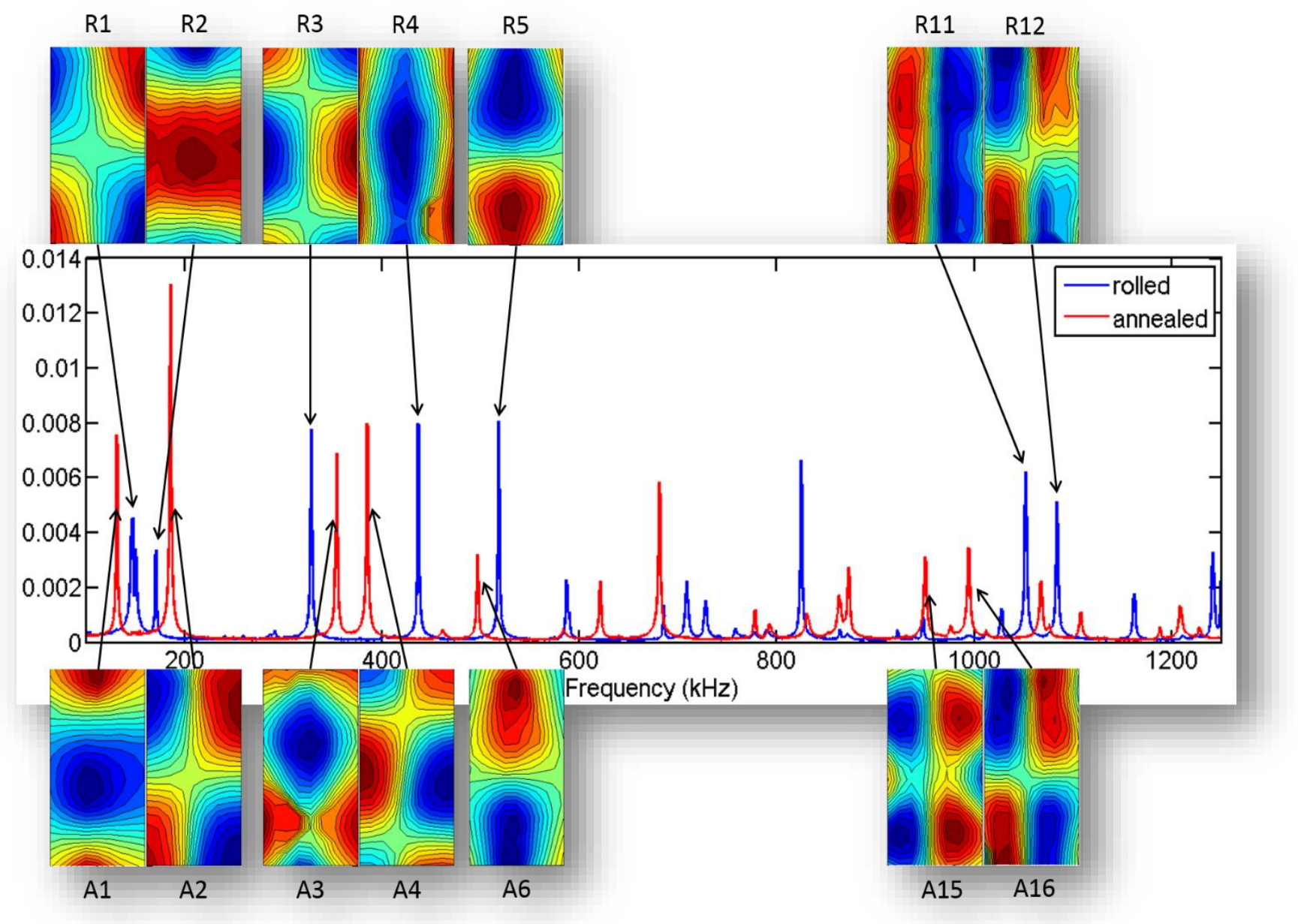

Figure 2. Resonant mode frequencies before (blue) and after (red) recrystallization. The insets show the resonant mode shapes before (R1 - R5, R11, R12) and after (A1 - A4, A6, A15, A16) recrystallization.

Mode frequency versus temperature is plotted in Figure 3. In the figure, modes R1 - R5 are tracked throughout the heating and cooling process. A similar tracking process was carried out for modes R11 and R12. Note how the modes change position relative to one another. Mode R1 becomes mode A2; mode R2 becomes mode A1. Mode R3 becomes A4; R4 becomes A3. Without knowledge of the mode shapes, one would tend to assume that each mode shifts down in frequency as it goes to the lower energy, annealed state. In reality, modes R1 and R3 increase in frequency. Simply tracking frequencies, without verifying mode shape, can lead to false conclusions. 

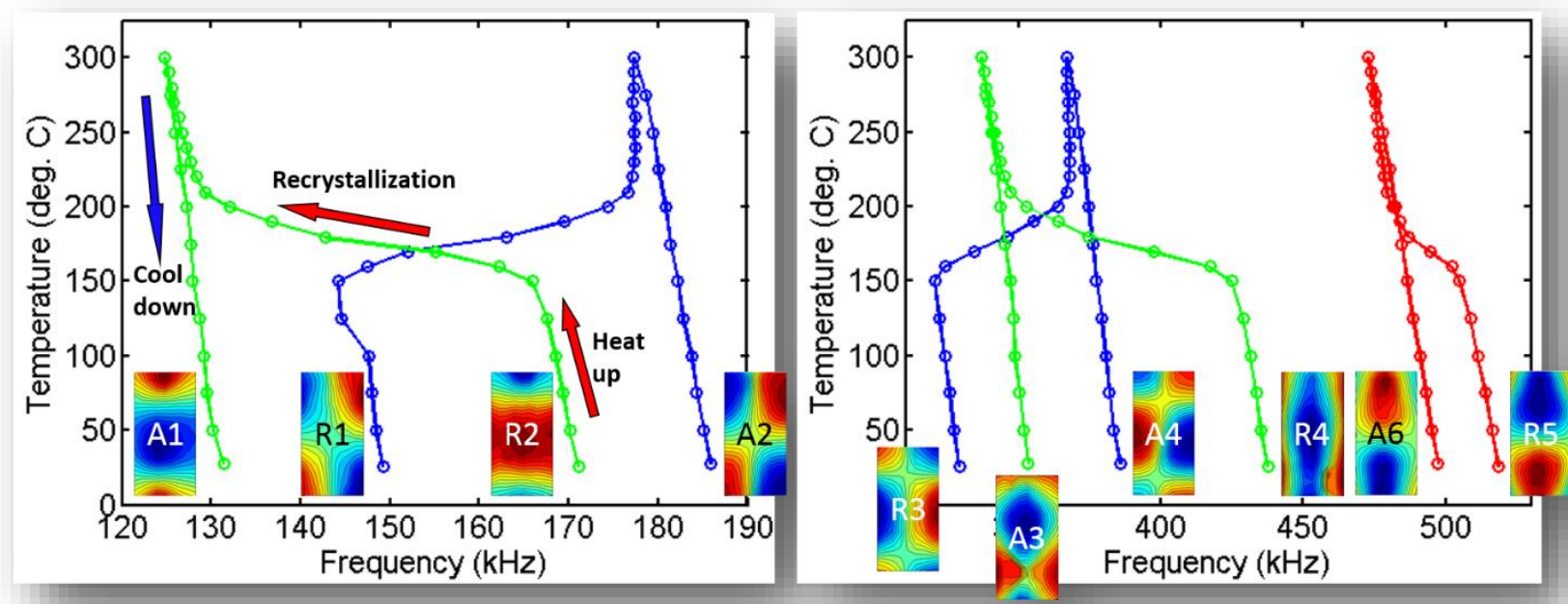

Figure 3. Tracks of individual modes throughout the heating and cooling process. Note how modes R1 and $\mathrm{R} 2$ and modes $\mathrm{R} 3$ and $\mathrm{R} 4$ change position relative to one another.

One peculiarity to note from Figures 2 and 3 is the shape of mode A3. It is notably asymmetric. The reason for the asymmetry is that this mode image is actually a concatenation of two modes that lie close together in frequency - so close that they produce a single peak in the frequency map shown in Figure 2. One of those modes is R4. The other mode does not lie close to R4 in the rolled condition, and thus, the single, symmetric mode shape can be ascertained.

\section{Path Forward}

This study demonstrates that the MPM is well suited to monitor grain restructuring of surrogate metallic fuels at high temperatures. Moving forward will involve transitioning the benchtop instrument into an instrument suitable for in-pile measurement of grain restructuring. There are both materials science and instrumentation issues to consider.

First consider the materials science issue. Due to the exorbitant cost and time involved, optimal design of in-pile experiments is crucial. A central part of the experimental design effort will involve modeling the expected experimental result. The materials science of grain restructuring is extremely complex and detailed computation models do not exist. The path forward here is to establish collaboration with Professor Anter El-Azab at Purdue University to work with Larry Aagesen at INL to develop a phase field model of recrystallization. This model will be informed with a continuum-based dislocation dynamics model.

Second consider the instrumentation issue. The measurement approach depends on the ability to transmit laser beams to and from the sample of interest using optical fibers. The survivability of the fibers is an issue that is being considered under another work package within the In-pile Instrumentation Initiative. Another instrumentation issue involves how to support the sample. Ideally for RUS experiments, the sample should have minimal contact with the environment. While this can be realized in the laboratory using acoustically mismatched ball bearings, this configuration is not realistic for in-pile measurement. Supporting the sample rigidly on one end (i.e. cantilever) would be a more tenable approach. The issue that will be considered in FY18 is how to accurately model the resonant response of a cantilever. 


\section{References}

${ }^{i}$ D. H. Hurley and J. B. Spicer, J. Appl. Phys., vol. 86, pp. 3423-3427, 1999.

ii D. H. Hurley, S. J. Reese, F. Farzbod, J. App. Phys., vol. 111, 53527, 2012. 